\title{
2 Umwelteinflüsse auf die männliche Fertilität
}

\author{
Hans-Christian Schuppe und Andreas Jung
}

\subsection{Vorbemerkungen}

Die Weltgesundheitsorganisation (WHO) subsumiert unter „reproduktiver Gesundheit“ die Fähigkeit von Frauen und Männern, ihre Fertilität zu kontrollieren und ihre Fortpflanzungswünsche unter Erhalt der eigenen Gesundheit, der ihrer Kinder sowie bei Zufriedenheit mit den sexuellen Beziehungen umzusetzen. In diesem Kontext wird als „Umwelt“ die Gesamtheit physikalischer, chemischer, biologischer, verhaltensbedingter und sozioökonomischer Faktoren und Verhältnisse definiert, die den menschlichen Organismus umgeben (Skakkebaek et al. 1991; Woodruff et al. 2008).

Angesichts der komplexen Entwicklung und Regulation des männlichen Reproduktionssystems ergeben sich sehr unterschiedliche Angriffspunkte für exogene Noxen:

- Endokrine Regulation der Hodenfunktion (Hypothalamus, Hypophyse)

- Androgenbiosynthese und -metabolismus

- Hormonrezeptoren (z.B. Androgen-, Östrogenrezeptoren)

- Spermatogenese (Hoden)

- Spermienreifung und -funktion (Nebenhoden)

- Emission/Ejakulation

- Sexualfunktionen (Libido, Erektion)

Eine zentrale Stellung nehmen hierbei die Keimzellen ein, aber auch Funktionsstörungen somatischer Zellen wie z.B. der Sertoli-Zellen in den Tubuli seminiferi oder der hormonproduzierenden Leydig-Zellen sowie immunkompetente Zellen im Interstitium des Hodens sind zu berücksichtigen. Neben Route, Dosis und Dauer ist insbesondere der altersbezogene Zeitpunkt einer Exposition gegenüber exogenen Noxen zu beachten: Intrauterin (Embryo, Fötus), Peri-/Postnatalphase, Pubertät (Einsetzen meiotischer Teilungen der Keimzellen), Erwachsenenalter. Beim erwachsenen Mann stellen Spermatogonien kurz vor Erreichen der Mitose, pachytäne Spermatozyten und frühe Spermatiden „Schwachstellen“ der Keimbahn dar, an denen Zellen bevorzugt 
zugrunde gehen (Hilscher 1985). Auch die Spermatidendifferenzierung kann gestört werden, während reife Spermien vergleichsweise widerstandsfähig gegenüber exogenen Noxen sind.

Zur Erfassung reproduktionstoxischer Effekte von Fremdstoffen, wie z.B. Pharmaka oder Industriechemikalien, wurden komplexe Tiermodelle etabliert, die sowohl Fruchtschäden als auch die Beeinträchtigung weiblicher und männlicher Fortpflanzungsfunktionen als Messparameter beinhalten (Spielmann 1992; Woodruff et al. 2008). Die Übertragbarkeit derartiger tierexperimenteller Befunde auf den Menschen ist jedoch limitiert, nicht zuletzt aufgrund erheblicher speziesspezifischer Unterschiede in der Spermatogenese. Darüber hinaus kommt der Verteilung und Metabolisierung von Fremdstoffen im Organismus sowie genetisch determinierten Unterschieden in der individuellen Suszeptibilität eine besondere Bedeutung zu (Schuppe et al. 2000).

Die Beeinträchtigung der männlichen Fertilität ist oft multifaktorieller Genese. Fertilität ist in ihrer Komplexität demnach nicht mittels eines „Markers“ messbar. Auch ein Parameter wie die Zeit bis zum Eintritt einer Schwangerschaft („time to pregnancy“) bei einem Paar mit Kinderwunsch ist problematisch, da diese nicht allein auf andrologische Faktoren bezogen werden kann. Die Untersuchung der reproduktiven Gesundheit des Mannes erfordert eine dementsprechend aufwendige Analytik, die Hormonbestimmungen, eine standardisierte Ejakulatanalyse, Tests zur Spermatozoenfunktion und -integrität, gegebenenfalls auch Hodenbiopsien einschließt (s. Kap. II.1). Hierbei erlauben einzelne Parameter weder eine definitive Beurteilung, noch lässt ihre Beeinflussung direkt auf eine spezifische Noxe schließen.

\subsection{Welche Umwelteinflüsse sind relevant?}

Zu den Noxen, die eine Beeinträchtigung der männlichen Fertilität verursachen können, zählen neben physikalischen Faktoren wie ionisierender Strahlung und Hitze vor allem chemische Substanzen wie Schwermetallverbindungen, Pestizide, bestimmte Lösungsmittel sowie Chlororganika und polyzyklische aromatische Kohlenwasserstoffe (Bonde 2006; Woodruff et al. 2008). Zahlreiche weitere Substanzen stehen zumindest im Verdacht, ein reproduktionstoxisches Potenzial zu entfalten. Hierbei sind nicht nur Umweltchemikalien und Berufsstoffe zu berücksichtigen, sondern auch Pharmaka einschließlich sog. Lifestyle-Medikamente, Rauschmittel sowie die klassischen Genussgifte Tabak und Alkohol (Schuppe et al. 2011).

\subsection{Physikalische Faktoren}

\subsubsection{Hitze}

Für einen ungestörten Ablauf der Spermatogenese ist beim Menschen eine Hodentemperatur erforderlich, die $1-2^{\circ} \mathrm{C}$ unterhalb der Körperkerntemperatur liegt. In Übereinstimmung mit tierexperimentellen Daten zeigte Watanabe (1959) bereits vor einem halben Jahrhundert für den Menschen, dass eine skrotale Exposition mit $45^{\circ} \mathrm{C}$ heißem Wasser für $1 / 2$ Stunde nach 12-maliger täglicher Anwendung zu einer ausgeprägten Verschlechterung der Samenqualität für 5-12 Wochen führt. Eine derartige geni- 
tale Hitzebelastung wurde in der ersten Hälfte des letzten Jahrhunderts besonders in Indien und Japan als Kontrazeptionsmethode propagiert. Entsprechend kommen regelmäßige heiße Vollbäder als Ursache für eine eingeschränkte Ejakulatqualität in Betracht (Jung u. Schuppe 2007).

Fieber führt zu Skrotaltemperaturen, die teilweise deutlich oberhalb von $37^{\circ} \mathrm{Cliegen}$. Folglich kann nach Fieberepisoden eine Verschlechterung der Ejakulatqualität bis hin zur Azoospermie für circa 4-12 Wochen eintreten (Jung et al. 2001; Carlsen et al. 2003). Bei einem Saunagang von 12 Minuten kommt es im Mittel zu einer Skrotaltemperatur von $37,5^{\circ} \mathrm{C}$ (Jockenhövel et al. 1990), überzeugende Studien zu einem möglichen Zusammenhang zwischen regelmäßigen Saunaaufenthalten und einer eingeschränkten Ejakulatqualität liegen jedoch nicht vor. Eine Erhöhung der Skrotaltemperaturen durch Windeln im Säuglings- und Kleinkindalter ist als klinisch nicht relevant einzustufen, da die besonders temperaturempfindlichen meiotischen und post-meiotischen Keimzellen zu diesem Zeitpunkt noch nicht im Hoden vorhanden sind (Jung u. Schuppe 2007).

Die Temperatur der Skrotalhaut korreliert gut mit der Hodentemperatur und lässt sich im Gegensatz zu dieser auch unter dynamischen Bedingungen einfach messen. Langdauernde Sitzphasen auf üblichen Stühlen führen zu Skrotaltemperaturen, die mit $36,4^{\circ} \mathrm{C}$ deutlich über den Werten für moderates Laufen mit $34,5^{\circ} \mathrm{C}$ liegen (Jung et al. 2005). Entsprechend erhöhte Werte finden sich auch während längerer Sitzphasen im Auto, bei Aktivierung der Sitzheizung ergibt sich eine zusätzliche genitale Temperaturbelastung von $\mathrm{ca} .0,5^{\circ} \mathrm{C}$ (Jung et al. 20o8). Eine vergleichbare Skrotaltemperatur-Erhöhung entsteht bei der Verwendung eines Laptop-Computers mit Positionierung auf den Oberschenkeln (Sheynkin et al. 2005). Moderates Fahrradfahren führt dagegen nicht zu einer wesentlichen Hitzebelastung (Jung u. Schuppe 2007).

In anderen Studien wurde eine Korrelation zwischen Skrotaltemperatur und Dauer der Berufstätigkeit in sitzender Position sowie eine negative Korrelation zwischen Skrotaltemperatur und Spermienkonzentration mit einem Abfall von 40\% pro Temperaturanstieg $\mathrm{um}^{\circ} \mathrm{C}$ beobachtet (Hjollund et al. 2002). Bei der Untersuchung fertiler Paare fand sich in der Cruppe der Berufskraftfahrer eine signifikant verlängerte Zeit bis zum Eintritt der Schwangerschaft (von 2,8 auf 4,5 Monate; Thonneau et al. 1997). Eine berufliche Hitzeexposition z.B. in der Stahlproduktion oder Keramikindustrie ist als potenziell gefährdend einzustufen, aussagekräftige Studien stehen hierzu jedoch nicht zur Verfügung (Jung u. Schuppe 2007).

\subsubsection{Ionisierende Strahlung}

Die Wirkung ionisierender Strahlen auf die Hodenfunktion ist gut dokumentiert (Rowley et al. 1974; Hoyes u. Morris 1996). In Abhängigkeit von Gesamtdosis und Fraktionierung kommt es zu einer Schädigung des Keimepithels, insbesondere der proliferierenden Spermatogonien. Hierbei verursacht eine fraktionierte Bestrahlung eine stärkere Schädigung als eine gleich hohe Einzeldosis. Mit einer vorübergehenden Oligozoospermie ist bereits nach einer kumulativen Dosis von o,1-o,3 Gy zu rechnen, die bei höheren Dosen auftretende Azoospemie ist nach Applikation von mehr als 3 Gy zumeist irreversibel (s. Tab. 1). Entsprechende Auswirkungen wurden nach Strahlenunfällen sowie Atombombenexplosionen beobachtet (Neel 1998). 
Tab. 1 Effekte ionisierender Strahlen auf die Hodenfunktion: Dosis-Wirkungsbeziehung (nach Rowley et al. 1974)

\begin{tabular}{llcc}
\hline Dosis (Gy) & Wirkung & FSH & Reversibilität \\
\hline 0,2 & Einzelne nekrotische Spermatogonien & $n-\uparrow$ & - \\
\hline 0,5 & Oligozoospermie & $\uparrow \uparrow$ & 6 Monate \\
\hline 0,78 & Azoospermie & $\uparrow \uparrow$ & 6 Monate \\
\hline 1,0 & Azoospermie & $\uparrow \uparrow$ & $9-18$ Monate \\
\hline $2,0-3,0$ & Azoospermie & $\uparrow \uparrow \uparrow$ & 30 Monate \\
\hline $4,0-6,0$ & Azoospermie & $\uparrow \uparrow \uparrow$ & $\geq 5$ lahre/keine \\
\hline
\end{tabular}

\subsubsection{Elektromagnetische Felder}

Der Einfluss von Mikrowellenstrahlung auf die männliche Fertilität ist belegt. Nach Exposition von Freiwilligen in kontrazeptiver Absicht mit Mikrowellen einer Frequenz von $2.450 \mathrm{MHz}$ über $30 \mathrm{~min}$ in dreiwöchigen Intervallen wurden die Probanden infertil, wobei dieser Effekt über intratestikuläre Temperaturen von $40-42^{\circ} \mathrm{C}$ während der Exposition zu erklären ist (Fang et al. 1982). Auch unter Alltagsbedingungen hat die Exposition gegenüber Radarwellen möglicherweise Auswirkungen auf die männliche Fertilität. In einer Fragebogen-Erhebung unter Angehörigen der Norwegischen Marine fand sich nach Adjustierung für Nikotinkonsum und andere Confounder in den Cruppen „Telekommunikation“ und „Radar/Sonar“ ein erhöhtes Risiko für Infertilität $(\mathrm{OR}=1.72 \mathrm{bzw} . \mathrm{OR}=2.28)$, nicht jedoch in der Gruppe „Elektronik“ (Møllerløkken u. Moen 2008).

Besonderes öffentliches Interesse gilt der mit der Benutzung von Mobiltelefonen einhergehenden elektromagnetischen Strahlung (Hochfrequenzfelder im $\mathrm{GHz}$-Bereich geringer Intensität). In einigen Studien konnten Korrelationen zwischen Nutzungsdauer (> 4 h/Tag im aktiven Modus) und Ejakulatqualität beobachtet werden, insbesondere eine Abnahme der Progressmotilität der Spermien (Agarwal et al. 2008; Fejes et al. 2008). Es ist jedoch nicht auszuschließen, dass Handy-unabhängige LifestyleFaktoren oder berufliche Umweltbelastungen zu den signifikanten Unterschieden zwischen den Gruppen mit geringfügigem bzw. intensivem Handy-Gebrauch führen. Im Niedrigfrequenzbereich der Elektrizitätsversorgung mit elektromagnetischen Feldern geringer Stärke, z.B. im privaten Umfeld, ist nach derzeitigem Wissensstand nicht von einem relevanten Gefährdungspotenzial auszugehen (Bonde 2006).

\subsection{Berufsstoffe und Umweltchemikalien}

Die Mehrzahl klinischer Beobachtungen über reproduktionstoxische Effekte von Fremdstoffen bezieht sich auf arbeitsplatzbedingte Expositionen (Tas et al. 1996; Bonde 2006; s. Tab. 2). Als klassisches Beispiel sei das in den 1970er-Jahren auf Bananenund anderen Obstplantagen eingesetzte Nematozid 1,2-Dibrom-3-chlorpropan (DBCP) genannt, das je nach Expositionsdauer eine irreversible Schädigung der Spermatogenese bis zur Azoospermie sowie eine Erhöhung der Gonadotropine verursacht (Whorton et al. 1977; Potashnik u. Porath 1995). Tierexperimentelle Befunde belegen, 
Tab. 2 Exogene Noxen für die männliche Fertilität: Berufsstoffe und Umweltchemikalien (Beispiele)*

\begin{tabular}{|l|l|}
\hline \multirow{2}{*}{$\begin{array}{l}\text { Schwermetalle } \\
\text { Pestizide, Herbizide }\end{array}$} & Beispiele \\
\hline $\begin{array}{l}\text { Lösungsmittel } \\
\text { Weichmacher }\end{array}$ & Dibromchlorpropan, Ethylendibromid \\
\hline Nichtionische Tenside & Glykolether, Kohlenstoffdisulfid \\
\hline Chlororganika & Phthalate \\
\hline Amide & Alkylphenole \\
\hline Synthetische Östrogene & DDT, Dioxine, polychlorierte Biphenyle \\
\hline * Zahlreiche potenziell fertilitätsschädigende Chemikalien gehören zu den „Endokrinen Disruptoren“, d.h. entfalten \\
\hline \begin{tabular}{l} 
östrogen-ähnliche, antiöstrogene oder antiandrogene Wirkungen. \\
\hline
\end{tabular}
\end{tabular}

dass DBCP seine spezifische, hodenschädigende Wirkung erst nach metabolischer Aktivierung zu einem hochreaktiven Episulfoniumion entfaltet. In Verlaufsstudien konnte demonstriert werden, dass bei einem Teil der Männer noch mehrere Jahre nach Beendigung der Exposition eine Regeneration der Spermatogenese einsetzen kann (Potashnik u. Porath 1995). Für weitere Pestizide wie z.B. Kepon, DDT und seine Metabolite oder 2,3,7,8-Tetrachlordibenzo-p-dioxin-haltige Mittel wird eine Beeinträchtigung der männlichen Fertilität diskutiert (Tas et al. 1996; Pflieger-Bruss et al. 2004).

Als relevante Schadstoffe wurden darüber hinaus Glykolether (z.B. 2-Ethoxyethanol) und Kohlenstoffdisulfid aus der Cruppe der organischen Lösungsmittel identifiziert (Tas et al. 1996; Veulemans et al. 1993). Ebenso gelten die Schwermetalle Blei und Quecksilber mit ihren Verbindungen als gesicherte Reproduktionstoxine (Bonde 2006). Vor Einführung entsprechender arbeitshygienischer Maßnahmen wurden schwerwiegende Hodenfunktionsstörungen nach Bleiintoxikationen beobachtet (Tas et al. 1996).

Sowohl ein fraglicher allgemeiner Abwärtstrend in der Spermaqualität als auch Hinweise auf eine Zunahme von Hodentumoren werden mit einer vermehrten Exposition gegenüber Fremdstoffen mit hormonähnlicher Wirkung in Zusammenhang gebracht (Aitken et al. 2004), wobei sich diese Hypothese vornehmlich auf tierexperimentelle Befunde in vivo und in vitro stützt. Neben Phyto- und Mykoöstrogenen können verschiedene Chemikalien aus Industrie und Umwelt, wie die bereits genannten Pestizide, polychlorierte Biphenyle (PCB), Dioxine oder Phthalate, östrogenähnliche, antiöstrogene oder antiandrogene Eigenschaften aufweisen und werden als „endocrine disruptors“ bezeichnet (Pflieger-Bruss et al. 2004). Die in der Umwelt persistenten chlorierten Kohlenwasserstoffe wie PCB konnten sowohl im männlichen als auch im weiblichen Genitaltrakt in relevanten Konzentrationen nachgewiesen werden. Im Hinblick auf „endocrine disruptors“ ist die Datenbasis für eine reproduktionstoxikologische Risikoabschätzung beim Menschen jedoch noch unzureichend, beispielsweise finden sich widersprüchliche epidemiologische Studien zum Einfluss von Phthalaten auf die männliche Fertilität (Duty et al. 2003; Herr et al. 2009). 


\subsection{Genussgifte}

\subsubsection{Tabakkonsum}

Die Einschränkung der Fertilität sowie die gesundheitliche Gefährdung der Nachkommen als Folge des Rauchens finden in der Bevölkerung zu wenig Beachtung. Nach neueren Schätzungen sind bei Paaren mit unerfülltem Kinderwunsch die zugrundeliegenden Fertilitätsstörungen in bis zu 13\% der Fälle auf den Nikotinkonsum zurückzuführen (The Practice Committee of the American Society for Reproductive Medicine 2008).

Zahlreiche Studien und Meta-Analysen belegen, dass sowohl die Chancen auf eine spontane Konzeption als auch die Erfolgsraten einer assistierten Reproduktion bei Raucherinnen gegenüber Nichtraucherinnen signifikant vermindert sind (Dechanet et al. 2011). Aufseiten des Mannes muss der Tabakkonsum ebenfalls als erheblicher Risikofaktor für eine reduzierte Fertilität angesehen werden; der formale Nachweis dieses Zusammenhangs ist jedoch schwierig, die Datenbasis entsprechend begrenzt (Schuppe et al. 2011). Negative Einflüsse des Rauchens auf die männliche Zeugungsfähigkeit wurden sowohl bei gesunden Probanden als auch bei Patienten mit gestörter Fertilität anhand der Untersuchung verschiedener Parameter dargestellt (s. Tab. 3). Im Vergleich zu Nichtrauchern ließ sich bei Rauchern eine moderate Abnahme von Spermienkonzentration, -gesamtzahl und -motilität beobachten, ebenso eine Verminderung des Anteils normal geformter Spermien (Vine et al. 1996; Künzle et al. 2003).

Außer den dargestellten negativen Effekten des Tabakkonsums, die unmittelbar die Spermien betreffen, wurde bei Rauchern auch über eine Beeinträchtigung der Funktion von Nebenhoden, Bläschendrüsen und Prostata berichtet, einhergehend mit einem verminderten Ejakulatvolumen, einer erniedrigten antioxidativen Kapazität des Seminalplasmas sowie Zunahme der Leukozyten und reaktiven Sauerstoffspezies (ROS) im Ejakulat (Saleh et al. 2002; Pasqualotto et al. 2008).

Angesichts der Vielzahl toxischer Inhaltsstoffe im Tabakrauch stellt sich auch die Frage nach einer Schädigung der DNA in menschlichen Spermien (s. Tab. 3). Untersuchungen zur DNA-Integrität bzw. -Fragmentation sowie bestimmter oxidativer Modifikationen der Spermien-DNA bei Rauchern und Nichtrauchern erbrachten allerdings kontroverse Ergebnisse (Schuppe et al. 2011).

Eine signifikante Reduktion der Konzeptionswahrscheinlichkeit pro Zyklus bzw. verlängerte Wartezeit bis zum Eintritt einer Schwangerschaft („Time to Pregnancy“, TTP) wird sowohl durch Rauchen der Frau als auch des Mannes verursacht (>15 Zigaretten pro Tag; Hassan u. Killick 2004). Ein negativer Einfluss des Tabakkonsums ist auch unter den Bedingungen der assistierten Reproduktion nachweisbar. Die Ergebnisse verschiedener Studien zeigen, dass die Schwangerschaftsraten nach In-vitroFertilisation (IVF) bei Partnerinnen von Rauchern gegenüber nichtrauchenden Paaren signifikant vermindert sind (Dechanet et al. 2011). Für Exraucher wurde eine langsame Normalisierung der Erfolgsaussichten einer ICSI-Behandlung über eine Abstinenzzeit von mindestens 2 Jahren berichtet.

Im Hinblick auf die Fertilität männlicher Nachkommen wurde der negative Einfluss der vorgeburtlichen Schadstoffbelastungen durch Rauchen der Mutter während der Schwangerschaft herausgestellt. Ein Konsum von mehr als 10 Zigaretten pro Tag war 
Tab. 3 Beeinträchtigung der männlichen Fertilität durch Tabakkonsum (Schuppe et al. 2011, mit freundlicher Genehmigung von Springer Science and Business Media)

\begin{tabular}{|c|c|}
\hline Sexualfunktionen & erektile Dysfunktion \\
\hline Ejakulatqualität & $\begin{array}{l}\text { Ejakulatvolumen } \downarrow \\
\text { Spermienkonzentration und -motilität } \downarrow \\
\text { Anteil pathomorpher Spermien } \uparrow \\
\text { Leukozyten \& Reaktive Sauerstoffspezies (ROS) } \uparrow \\
\text { Antioxidative Kapazität } \downarrow\end{array}$ \\
\hline Spermienstruktur und -funktion & $\begin{array}{l}\text { DNA-Schäden einschließlich Benzpyren-assoziierter DNA-Addukte; } \\
\text { Disomie-Rate } \uparrow \\
\text { Beeinträchtigung der Chromatinkondensation } \\
\text { Störung der axonemalen Ultrastruktur } \\
\text { Akrosinaktivität } \downarrow \\
\text { Induzierbarkeit der Akrosomreaktion } \downarrow\end{array}$ \\
\hline Schwangerschaft & $\begin{array}{l}\text { verminderte Schwangerschaftsraten bei } \\
\text { Konzeption in vivo } \\
\text { konventioneller In-vitro-Fertilisation } \\
\text { intrazytoplasmatischer Spermieninjektion } \\
\text { erhöhte Abortrate }\end{array}$ \\
\hline Nachkommen & $\begin{array}{l}\text { Nachweis Benzpyren-assoziierter DNA-Addukte in Embryonen }{ }^{1} \\
\text { Risiko eines plötzlichen Kindstodes } \uparrow \\
\text { maligne Erkrankungen } \uparrow^{1} \\
\text { gestörte Fertilität der Söhne }{ }^{2}\end{array}$ \\
\hline
\end{tabular}

mit einer signifikant niedrigeren Spermienkonzentration der Söhne im Vergleich zu Nachkommen nichtrauchender Frauen assoziiert (Ramlau-Hansen et al. 2007).

Aufseiten des Mannes sind neben der Schädigung der Gameten infolge des Tabakkonsums auch negative Einflüsse auf Sexualfunktionen als Ursache verminderter Chancen auf eine spontane Konzeption zu beachten (s. Tab. 3). Zigarettenrauchen ist ein erheblicher Risikofaktor für die Entwicklung von Erektionsstörungen als Ausdruck einer vaskulären Schädigung.

\subsubsection{Alkohol}

Bei chronischem Alkoholmissbrauch kommt es nicht selten zu gravierenden Störungen des männlichen Reproduktionssystems (Schuppe et al. 2011). Zu den bekannten Symptomen des Hypogonadismus (s. Kap. II.11) infolge einer alkoholinduzierten Leberinsuffizienz gehören Gynäkomastie, Hodenatrophie mit entsprechender Einschränkung der Spermatogenese und Infertilität, erektile Dysfunktion sowie Libidoverlust. Zusätzlich sind indirekte negative Auswirkungen der mit der Alkoholabhängigkeit verbundenen Mangelernährung zu berücksichtigen. 
Verschiedene Studien belegen eine Beeinträchtigung der Ejakulatqualität mit vermindertem Volumen, herabgesetzter Spermienkonzentration und -motilität sowie Zunahme pathomorpher Spermien. Schwerwiegende Folge eines exzessiven Konsums ist die Alkohol-induzierte Orchipathie mit Auflösung des Keimepithelverbandes in den Samenkanälchen bis hin zu einem Sertoli-cell-only-Syndrom (Pajarinen u. Karhunen 1994).

Mehrere Studien sprechen dafür, dass ein moderater Alkoholkonsum des Mannes nicht zu einer Verschlechterung der Ejakulatparameter bzw. Subfertilität führt. Im Zusammenhang mit riskantem Alkoholkonsum des Mannes (> 20 Drinks pro Woche) erscheint dagegen der Zeitraum bis zum Eintritt einer Schwangerschaft (,time-topregnancy“) signifikant verlängert (Hassan u. Killick 2004). Auch in einem IVF-Programm wurde Alkoholkonsum des Mannes bzw. der Frau ebenfalls als negativer Einflussfaktor im Hinblick auf die Schwangerschaftsraten identifiziert (Klonoff-Cohen et al. 2003).

\subsubsection{Koffein}

Koffein gehört weltweit zu den am häufigsten konsumierten pharmakologisch aktiven Substanzen. Als Quellen sind nicht nur Kaffee und Tee, sondern auch alkoholfreie Getränke, „Alcopops“, Kakao, Schokolade sowie Medikamente zu berücksichtigen. In vitro stimuliert Koffein ähnlich wie Pentoxifyllin die Motilität humaner Spermien. Im Zusammenhang mit Kaffeegenuss wurde bei Männern aus einer Kinderwunschsprechstunde eine Zunahme der Konzentration motiler Spermien im Ejakulat beobachtet, bei gleichzeitigem Konsum von über 4 Tassen Kaffee und mehr als 20 Zigaretten pro Tag fand sich allerdings eine verminderte Spermienmotilität und -vitalität (Marshburn et al. 1989). In anderen Studien zeigte sich keine Assoziation zwischen Koffeinaufnahme des Mannes und Ejakulatqualität bzw. Konzeptionswahrscheinlichkeit (Hassan u. Killick 2004).

\subsection{Arzneimittel und illegale Drogen}

Unter den exogenen Noxen, die einen negativen Einfluss auf verschiedene Funktionen des männlichen Reproduktionssystems haben können, sind auch Pharmaka zu berücksichtigen (Krause 2008).

Wie bereits oben erwähnt, ergeben sich vielfältige Angriffspunkte im männlichen Reproduktionssystem:

- Störungen der endokrinen Regulation (z.B. Sexualhormone, Glukokortikoide)

- Störungen der Androgenbiosynthese (z.B. Ketoconazol)

- Störungen des Testosteronmetabolismus (z.B. Antikonvulsiva)

- Androgenrezeptor-Antagonisten (z.B. Spironolacton)

- Störungen der Spermatogenese (z.B. Zytostatika)

- Störungen der Spermatozoenfunktion (z.B. Antibiotika [in vitro])

- Störungen der Emission/Ejakulation (z.B. Antidepressiva, psychotrop wirksame Medikamente)

- Störungen der Erektion (z.B. Antihypertensiva)

- Störungen der Libido (z.B. Benzodiazepine) 
Neben ärztlich verordneten Präparaten ist eine hohe Rate an Eigenmedikationen zu beachten: In einer Studie gaben $77 \%$ der Paare bei der Erstuntersuchung wegen unerfülltem Kinderwunsch die Einnahme frei käuflicher Arzneimittel an (Olatunbosun et al. 1997). Darüber hinaus spielt mit Blick auf die verfügbaren epidemiologischen Daten der Abusus leistungssteigernder Pharmaka, vor allem anabol-androgener Steroide (AAS), sowie illegaler Drogen eine bedeutsame Rolle (s. Kap. II.18, II.19).

Die Datenbasis bezüglich der Wirkung illegaler Drogen wie Cannabis (Marijuana, Haschisch), Kokain oder Opiaten auf die reproduktiven Funktionen des Mannes ist sehr begrenzt (Schuppe et al. 2011). Inwieweit Cannabis, die mit Abstand am häufigsten konsumierte illegale Droge, dosisabhängig zu einer Erniedrigung des Serumtestosteronspiegels und der Ejakulatqualität führt, wurde in früheren Studien kontrovers beurteilt. Andererseits spricht das vermehrte Auftreten einer Gynäkomastie bei Cannabiskonsum für eine Störung der Androgen-Östrogen-Balance (s. Kap. II.12).

Kokainkonsum ist ebenfalls mit einem erhöhten Risiko für eine eingeschränkte Ejakulatqualität (Spermienkonzentration, -motilität) assoziiert; gesicherte Daten zum Einfluss auf die männliche Fertilität liegen jedoch nicht vor. Im Zusammenhang mit Opiaten wie Morphin, Heroin oder Methadon ist vor allem die Hemmung der $\mathrm{CnRH}-$ Sekretion über eine Aktivierung opiaterger Efferenzen im Hypothalamus und damit das klinische Bild eines hypogonadotropen Hypogonadismus zu beachten. Entsprechend geben männliche Heroinabhängige häufig Störungen der Sexualfunktionen an. Daneben wurden über eine Beeinträchtigung der Spermienmotilität sowie eine Zunahme pathologisch geformter Spermien durch Heroin und auch Methadon berichtet (Ragni et al. 1988). Daten über den Einfluss psychoaktiver Substanzen wie Amphetaminen einschließlich „Ecstasy“ (n-Methyl-3,4-Methylendioxymethamphetamin, MDMA), Benzodiazepinen oder synthetischen Halluzinogenen auf die männliche reproduktive Funktion fehlen weitgehend. In erster Linie wird auch hier über Störungen der Sexualfunktionen berichtet.

\subsection{Praktische Aspekte}

- In der täglichen Praxis sollte im Zusammenhang mit Störungen der männlichen Fertilität stets nach möglichen exogenen Noxen einschließlich Genussgiften und sog. Lifestyle-Medikamenten gefahndet werden.

- Angesichts der möglichen Spätfolgen von Tabak-, Alkohol- und Drogenkonsum für die reproduktive Gesundheit des Mannes ist die primäre Prävention als dringende ärztliche Aufgaben anzusehen.

- Kumulative Effekte verschiedener Noxen sind wahrscheinlich (s. Abb. 1), sowohl im Hinblick auf die Chancen einer Konzeption auf natürlichem Weg als auch die Erfolgsaussichten assistierter Reproduktionsverfahren.

- Fertilitätsstörungen des Mannes sind häufig multifaktorieller Genese; beispielsweise kann es bei einer bereits bestehenden Schädigung der Reproduktionsorgane erst durch Einwirkung zusätzlicher exogener Noxen zu einer klinisch manifesten Beeinträchtigung der Fertilität kommen. Die Beratung über gesundheitliche Auswirkungen und konsequente Elimination relevanter Noxen erlaubt z.T. beachtliche Verbesserungen der Ejakulatqualität, auch wenn diese lediglich als Co-Faktoren in Verdacht stehen. 


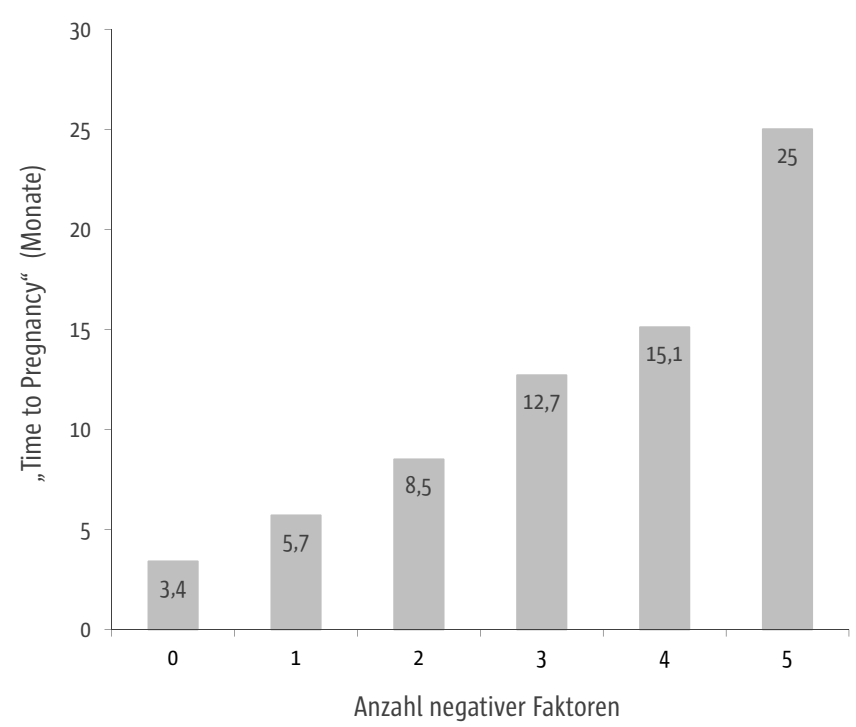

Abb. 1 Kumulative Effekte negativer Lifestyle-Faktoren auf die Fekundität: Zeit bis zum Eintritt einer Schwangerschaft („Time to Pregnancy“) (mod. nach Hassan u. Killic 2004) Fragebogenbasierte Studie an 2.112 schwangeren Frauen. Lifestyle-Faktoren: Frau oder Mann $>15$ Zigaretten/d; Mann > 20 alkoholische „Drinks“/Woche; Body mass index (BMI) der Frau $>25$ kg/m²; Kaffee- und/oder Teekonsum der Frau > 6 Tassen/d; Alter Frau > 35 lahre, Mann $>45$ lahre; sozialer Status. Signifikante Unterschiede zwischen allen Kategorien; $p<0.01$.

- Die andrologische Labordiagnostik, die neben Hormonbestimmungen und einer standardisierten Ejakulatuntersuchung nach WHO-Empfehlungen Tests zur Erfassung der Spermienfunktion und DNA-Integrität umfassen sollte (s. Kap. II.1), lässt nicht direkt auf eine spezifische Noxe schließen.

- Wichtigstes Hilfsmittel ist nach wie vor eine ausführliche Anamneseerhebung, die mögliche Expositionsrisiken in Beruf und Haushalt, bei Hobbies oder Freizeitaktivitäten sowie Lebensgewohnheiten erfasst.

- Die Abschätzung der Exposition gegenüber Gefahrstoffen am Arbeitsplatz erfordert gegebenenfalls die Zusammenarbeit mit betriebsärztlichen Diensten (Kenntnis der individuellen Arbeitsabläufe, Schutzmaßnahmen, durchgeführte Kontrolluntersuchungen). Ergänzend kann ein Biomonitoring, d.h. die Bestimmung der Konzentration eines Gefahrstoffes oder seiner Metaboliten in Körperflüssigkeiten, zur Abschätzung der tatsächlichen Exposition herangezogen werden. Allein der Nachweis eines Stoffes im Organismus belegt im Einzelfall jedoch nicht seine reproduktionstoxische Wirkung.

\section{Literatur}

Agarwal A, Deepinder F, Sharma RK, Ranga G, Li I (2008) Effect of cell phone usage on semen analysis in men attending infertility clinic: an observational study. Fertil Steril 89, 124-128

Aitken RJ, Koopman P, Lewis SE (2004) Seeds of concern. Nature 432, 48-52

Bonde JP (2006) Effects of lifestyle and toxicants. In: Schill W-B, Comhaire F, Hargreave TB (eds.) Andrology for the Clinician, Springer, Heidelberg, 348-357 
Carlsen E, Andersson AM, Petersen JH, Skakkebaek NE (2003) History of febrile illness and variation in semen quality. Hum Reprod 18, 2089-2092

Dechanet C, Anahory T, Mathieu Daude IC, Quantin X, Reyftmann L, Hamamah S, Hedon B, Dechaud H (2011) Effects of cigarette smoking on reproduction. Hum Reprod Update 17, 76-95

Duty SM, Silva MI, Barr DB, Brock JW, Ryan L, Chen Z, Herrick RF, Christiani DC, Hauser R (2003) Phthalate exposure and human semen parameters. Epidemiology 14, 269-277

Fang B, Lu Q, Li F, Zou R, Liu Y (1982) Application of microwave in male contraception. Chin J Urol 3, 75-78

Fejes I, Závaczki Z, Szöllsi |, Koloszár S, Daru I, Kovács L, Pál A (2008) Is there a relationship between cell phone use and semen quality? Systems Biology in Reproductive Medicine 51, 385-393

Hassan MAM, Killick SR (2004) Negative lifestyle is associated with a significant reduction in fecundity. Fertil Steril 81, 384-392

Herr C, zur Nieden A, Koch HM, Schuppe H-C, Fieber C, Angerer J, Eikmann T, Stilianakis NI (2009) Urinary di(2-ethylhexyl) phthalate (DEHP)-metabolites and human male markers of reproductive function. Int I Hyg Environ Health 212, 648-653

Hilscher W (1985) Umwelt und Fertilität - ein Beitrag zur Sensibilität der männlichen Keimbahn gegenüber Umweltnoxen. Z Hautkr 60, 1040-1065

Hjollund NHI, Storgaard L, Ernst E, Bonde JP, Olsen I (2002) Impact of diurnal scrotal temperature and semen quality. Reproductive Toxicology 16, 215-221

Hoyes KP, Morris ID (1996) Environmental radiation and male reproduction. Int | Androl 19, 199-204

Jung A, Leonhardt F, Schill W-B, Schuppe H-C (2005) Influence of the type of undertrousers and physical activity on scrotal temperature Hum Reprod 20, 1022-1027

Jung A, Schuppe H-C (2007) Influence of genital heat stress on semen quality in humans. Andrologia 39, 203-215

Jung A, Schuppe H-C, Schill W-B (2001) Fieber als Ursache einer temporären Fertilitäts-einschränkung des Mannes. Hautarzt 52, 1090-1093

Jung A, Strauß P, Lindner H-J, Schuppe H-C (2008) Influence of heating car seats on scrotal temperature. Fertil Steril 90, 335-339

Jockenhövel F, Gräwe A, Nieschlag E (1990) A portable digital data recorder for long-term monitoring of scrotal temperatures. Fertil Steril 54, 694-700

Klonoff-Cohen H, Lam-Kruglick P, Gonzalez C (2003) Effects of maternal and paternal alcohol consumption on the success rates of in vitro fertilization and gamete intrafallopian transfer. Fertil Steril 79, 330-339

Krause WKH (2008) Drugs compromising male sexual health. Springer, Berlin, Heidelberg, New York, Tokyo

Künzle R, Mueller MD, Hanggi W, Birkhauser MH, Drescher H, Bersinger NA (2003) Semen quality of male smokers and nonsmokers in infertile couples. Fertil Steril 79, 287-291

Marshburn PB, Sloan CS, Hammond MG (1989) Semen quality and association with coffee drinking, cigarette smoking, and ethanol consumption. Fertil Steril 52, 162-165

Møllerløkken 0|, Moen BE (2008) Is fertility reduced among men exposed to radiofrequency fields in the Norwegian navy? Bioelectromagnetics 29, 345-352

Neel JV (1998) Reappraisal of studies concerning the genetic effects of the radiation of humans, mice, and Drosophila. Environ Mol Mutagen 31, 4-10

Olatunbosun OA, Edouard L, Pierson RA (1997) How important is health promotion in the lifestyle of infertile couples? Clin Exp Obstet Gynecol 24, 183-186

Pajarinen J, Karhunen PI (1994) Spermatogenic arrest and "Sertoli cell-only“ syndrome - common alcohol-induced disorders of the human testis. Int | Androl 17, 292-299

Pasqualotto FF, Umezu FM, Salvador M, Borges E Ir, Sobreiro BP, Pasqualotto EB (2008) Effect of cigarette smoking on antioxidant levels and presence of leukocytospermia in infertile men: a prospective study. Fertil Steril 90, 278-283

Pflieger-Bruss S, Schuppe H-C, Schill W-B (2004) The male reproductive system and its susceptibility to endocrine disrupting chemicals. Andrologia 36, 337-345

Potashnik G, Porath A (1995) Dibromochloropropane (DBCP): a 17-year reassessment of testicular function and reproductive performance. I Occup Environ Med 37, 1278-1291

Ragni G, De Lauretis L, Bestetti 0, Sghedoni D, Gambaro V (1988) Gonadal function in male heroin and methadone addicts. Int | Androl 11, 93-100 
Ramlau-Hansen CH, Thulstrup AM, Storgaard L, Toft G, Olsen I, Bonde JP (2007) Is prenatal exposure to tobacco smoking a cause of poor semen quality? A follow-up study. Am | Epidemiol 165, 1372-1379

Rowley MI, Leach DR, Warner GA, Heller CG (1974) Effect of graded doses of ionizing radiation on the human testis. Radiat Res 59, 665-678

Saleh RA, Agarwal A, Sharma RK, Nelson DR, Thomas AJ (2002) Effect of cigarette smoking on levels of seminal oxidative stress in infertile men: a prospective study. Fertil Steril 78, 491-499

Schuppe H-C, Jung A, Köhn F-M, Haidl G (2011) Wie Genußgifte die Fertilität beeinflussen können. MMW-Fortschr Med 153, 33-36

Schuppe H-C, Wieneke P, Fritsche E, Donat S, Köhn F-M, Abel I (2000) Xenobiotic metabolism, genetic polymorphisms and male infertility. Andrologia 32, 255-262

Sheynkin Y, Jung M, Yoo P, Schulsinger D, Komaroff E (2005) Increase in scrotal temperature in laptop computer users. Human Reprod 2, 452-455

Skakkebaek NE, Negro-Vilar A, Michal F, Fathalla M (1991) Impact of the environment on reproductive health. Dan Med Bull 38, 425-426

Spielmann H (1992) Reproduktionstoxikologie: Oogenese und frühe embryonale Entwicklung. Fertilität 8, 100106

Tas S, Lauwerys R, Lison D (1996) Occupational hazards for the male reproductive system. Crit Rev Toxicol 26, 261-307

The Practice Committee of the American Society for Reproductive Medicine (2008) Smoking and Infertility. Fertil Steril 90, S254-259

Thonneau P, Ducot B, Bujan L, Mieusset R, Spira A (1997) Effect of male occupational heat exposure on time to pregnancy. Int | Androl 20, 274-278

Veulemans H, Steeno O, Masschelein R, Groeseneken D (1993) Exposure to ethylene glycol ethers and spermatogenic disorders in man: a case-control study. $\mathrm{Br}$ I Ind Med 50, 71-78

Vine MF, Tse CKI, Hu PC, Truong KY (1996) Cigarette smoking and semen quality. Fertil Steril 65, 835-842

Watanabe A (1959) The effect of heat on the human spermatogenesis. Kyushu I Med Sci 10, 101-117

Whorton D, Krauss RM, Marshall S, Milby TH (1977) Infertility in male pesticide workers. Lancet ii, 1259-1261

Woodruff TJ, Carlson A, Schwartz JM, Giudice LC (2008) Proceedings of the Summit on Environmental Challenges to Reproductive Health and Fertility: executive summary. Fertil Steril 89, 281-300 